\title{
Advances in the Direct Study of Carbon Burning in Massive Stars
}

\author{
G. Fruet, ${ }^{1,2}$ S. Courtin, ${ }^{1,2,3, *}$ M. Heine $\odot,{ }^{1,2, \dagger}$ D. G. Jenkins, ${ }^{4}$ P. Adsley, ${ }^{5}$ A. Brown, ${ }^{4}$ R. Canavan, ${ }^{6,7}$ W. N. Catford ${ }^{6}$ \\ E. Charon ${ }^{8}$ D. Curien, ${ }^{1,2}$ S. Della Negra, ${ }^{5}$ J. Duprat,${ }^{9}$ F. Hammache, ${ }^{5}$ J. Lesrel, ${ }^{5}$ G. Lotay, ${ }^{6}$ A. Meyer, ${ }^{5}$ D. Montanari, ${ }^{1,2,3}$ \\ L. Morris, ${ }^{4}$ M. Moukaddam, ${ }^{6}$ J. Nippert, ${ }^{1,2}$ Zs. Podolyák, ${ }^{6}$ P. H. Regan, ${ }^{6,7}$ I. Ribaud, ${ }^{5}$ M. Richer, ${ }^{1,2}$ M. Rudigier, ${ }^{6}$ \\ R. Shearman, ${ }^{6,7}$ N. de Séréville, ${ }^{5}$ and C. Stodel ${ }^{10}$ \\ ${ }^{1}$ IPHC, Université de Strasbourg, Strasbourg F-67037, France \\ ${ }^{2}$ CNRS, UMR7178, Strasbourg F-67037, France \\ ${ }^{3}$ USIAS/Université de Strasbourg, Strasbourg F-67083, France \\ ${ }^{4}$ University of York, York YO10 5DD, United Kingdom \\ ${ }^{5}$ Institut de Physique Nucléaire, CNRS/IN2P3, Université Paris-Sud, Université Paris-Saclay, 91406 Orsay Cedex, France \\ ${ }^{6}$ Department of Physics, University of Surrey, Guildford GU2 7XH, United Kingdom \\ ${ }^{7}$ National Physical Laboratory, Teddington, Middlesex TW110 LW, United Kingdom \\ ${ }^{8}$ NIMBE, CEA, CNRS, Université Paris-Saclay, CEA Saclay F-91191 Gif sur Yvette, France \\ ${ }^{9}$ Centre de Sciences Nucléaires et de Sciences de la Matière (CSNSM), Université Paris Sud, \\ UMR 8609-CNRS/IN2P3, 91405 Orsay, France \\ ${ }^{10}$ GANIL, CEA/DSM-CNRS/IN2P3, Caen F-14076, France
}

(Received 30 July 2019; revised manuscript received 27 September 2019; accepted 21 February 2020; published 12 May 2020)

\begin{abstract}
The ${ }^{12} \mathrm{C}+{ }^{12} \mathrm{C}$ fusion reaction plays a critical role in the evolution of massive stars and also strongly impacts various explosive astrophysical scenarios. The presence of resonances in this reaction at energies around and below the Coulomb barrier makes it impossible to carry out a simple extrapolation down to the Gamow window-the energy regime relevant to carbon burning in massive stars. The ${ }^{12} \mathrm{C}+{ }^{12} \mathrm{C}$ system forms a unique laboratory for challenging the contemporary picture of deep sub-barrier fusion (possible sub-barrier hindrance) and its interplay with nuclear structure (sub-barrier resonances). Here, we show that direct measurements of the ${ }^{12} \mathrm{C}+{ }^{12} \mathrm{C}$ fusion cross section may be made into the Gamow window using an advanced particle-gamma coincidence technique. The sensitivity of this technique effectively removes ambiguities in existing measurements made with gamma ray or charged-particle detection alone. The present cross-section data span over 8 orders of magnitude and support the fusion-hindrance model at deep sub-barrier energies.
\end{abstract}

DOI: 10.1103/PhysRevLett.124.192701

Introduction.-The number of fusion reactions that are critical for astrophysics is scarce. Among these, the ${ }^{12} \mathrm{C}+{ }^{12} \mathrm{C}$ reaction that is essential for the life cycle of massive stars, may occur at different stages of stellar evolution: explosive scenarios like Type Ia supernovae [1] which can be used as cosmological standard candles, quiescent carbon burning in the contracting core of a massive star [2,3] at temperatures of the order of $1 \mathrm{GK}$, and densities above a million $\mathrm{g} / \mathrm{cm}^{3}$ and possibly in superbursts of x-ray binary systems [4].

The obstacle to a reliable extrapolation of the ${ }^{12} \mathrm{C}+{ }^{12} \mathrm{C}$ cross section into the astrophysically relevant Gamow energy window is the presence of resonances in the cross section around the Coulomb barrier that continue down to the lowest collision energies accessible experimentally. This behavior is strikingly different from the smooth variation in cross section as a function of energy typical of fusion in other heavy-ion systems.

The presence of resonances in the ${ }^{12} \mathrm{C}+{ }^{12} \mathrm{C}$ reaction has been hotly debated for over 60 years. The conventional wisdom is that they correspond to the formation of shortlived molecular states $[5,6]$, and this early suggestion has led on to far wider discussion of clustering in alphaconjugate systems [7-12]. However, this model remains controversial: an alternate picture [13] is that the resonant behavior is simply an artifact of the low level density of the ${ }^{12} \mathrm{C}+{ }^{12} \mathrm{C}$ compound system. The general trend in the reaction cross section, masked by the presence of the individual resonances, should select sub-barrier hindrance, as widely observed in other heavy-ion systems $[14,15]$.

Given this controversy and the lack of a clear theoretical picture for the resonant behavior, the main approach from the experimental side has been to perform direct measurements of the cross section to the lowest energies possible. Recently, an alternative indirect approach using the so-called "Trojan horse" method (THM) has reported strong resonances relevant to ${ }^{12} \mathrm{C}+{ }^{12} \mathrm{C}$ fusion corresponding to very low collision energies within and below the Gamow window for massive stars [16]. Results of this Letter, i.e., a substantial increase of the $S$ factor 
in the Gamow window have been discussed by Mukhamedzhanov et al. [17] in terms of an artifact from using an invalid plane-wave approximation. Reliable direct data as obtained in the present work are essential to normalize indirect studies, but also to shed light on the present controversy regarding indirect methods.

At the lowest collision energies, ${ }^{12} \mathrm{C}+{ }^{12} \mathrm{C}$ fusion mainly leads to two final systems: ${ }^{23} \mathrm{Na}+p$ and ${ }^{20} \mathrm{Ne}+\alpha$. This immediately suggests two techniques for determining the cross section of the ${ }^{12} \mathrm{C}+{ }^{12} \mathrm{C}$ fusion reaction, namely, (i) detection of evaporated charged particles (protons or $\alpha$ ) and (ii) detection of gamma rays from excited states of ${ }^{23} \mathrm{Na}$ and ${ }^{20} \mathrm{Ne}$. Both techniques have been extensively employed showing consistent results at higher energies but exhibiting inconsistencies at lower energies [18-20], suggesting that systematic effects are a significant limitation [21,22]. An approach, which provides a unique signature and which can circumvent these experimental limitations, is to detect evaporated charged particles and gamma rays in coincidence. This effectively removes ambiguities such as those associated with protons created in reactions on target contaminants. Jiang et al. have pioneered this approach using a large array of high-purity germanium detectors, GAMMASPHERE, coupled to an array of annular silicon strip detectors to record the evaporated charged particles [23]. Their initial results were consistent with the results of earlier measurements using conventional techniques. However, the limitations of available beam time (a few days) and beam current (100s of pnA) did not allow them to push towards the astrophysically relevant energy region for ${ }^{12} \mathrm{C}+{ }^{12} \mathrm{C}$ fusion.

Experiment.-Here, we report on measurements of ${ }^{12} \mathrm{C}$ fusion well into the Gamow window relevant to the most massive stars $\left(M_{\odot} \approx 25\right)$ in the energy regime $E_{\text {beam }}=2.2$ to $5.4 \mathrm{MeV}$ (in the center-of-mass system) with the STELLA apparatus [24] for coincident gamma-particle detection. STELLA (see Fig. 1) was mounted on a dedicated beam line at the Andromède accelerator facility [25] at IPN Orsay, France. The intensity of the ${ }^{12} \mathrm{C}\left(2^{+} / 3^{+}\right)$ beam was increased from $30 \mathrm{pnA}$ around the Coulomb barrrier $\left(E_{\text {beam }}=6.6 \mathrm{MeV}\right)$ to $2 \mathrm{p} \mu \mathrm{A}$ for the astrophysically relevant region with data-taking periods of weeks.

STELLA comprises an ultrahigh vacuum chamber $\left(\approx 10^{-8}\right.$ mbar $)$ containing a rotating target mechanism that supports large diameter $(\approx 5 \mathrm{~cm})$ thin $(\approx 200 \mathrm{~nm})$ natural carbon foils which can be rotated at up to $1000 \mathrm{rpm}$ to efficiently dissipate heat from the intense ${ }^{12} \mathrm{C}$ beams and hence, prevent target deterioration. Continuous measurements of scattered beam at $45^{\circ}$ as well as foil-thickness and homogeneity measurements after irradiation confirm that carbon buildup in the target $[21,26]$ is below $1 \%$ and thus negligible. Samples of material from both irradiated and nonirradiated areas of some of the target foils were analyzed by Raman spectrometry to determine possible changes in the graphite, disordered carbon, and amorphous

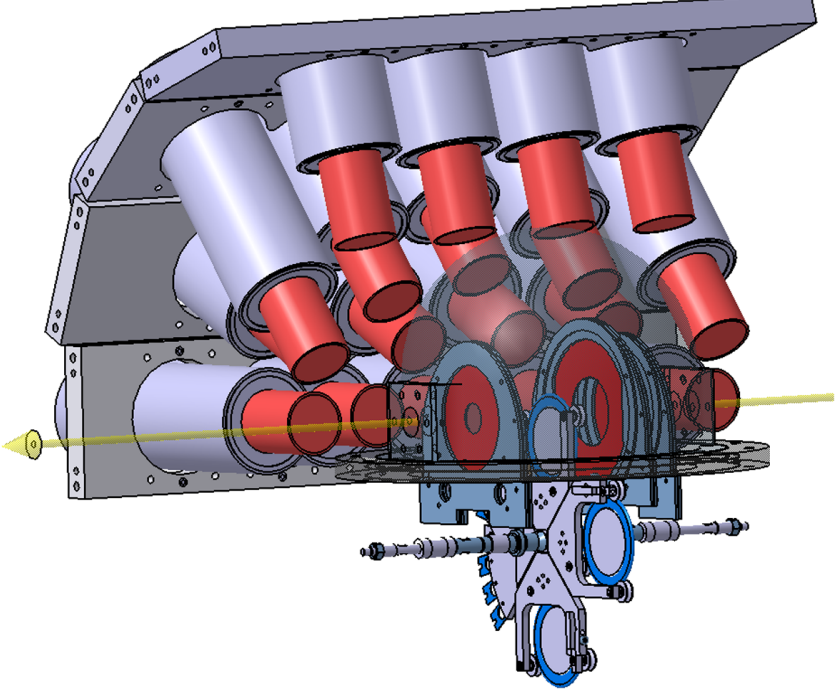

FIG. 1. 3D-rendered CAD drawing of the STELLA apparatus: The volume of the scattering chamber is shown semitransparent in order to allow its interior to be viewed, comprising three annular silicon detectors, and a target holding or rotating mechanism. The large circular carbon target foils are shown with blue frames on their perimeter. The direction of the ${ }^{12} \mathrm{C}$ beam (from right to left in the diagram) is shown with the yellow arrow. Only half of the $\mathrm{LaBr}_{3}(\mathrm{Ce})$ gamma-ray array is depicted for clarity of presentation.

carbon signatures [27]. Single crystal graphite is characterized by a single band at $1581 \mathrm{~cm}^{-1}$ referred to as the $G$ band. Amorphous carbon has a single broad and asymmetrical band centered at about $1520 \mathrm{~cm}^{-1}$ and disordered carbon usually shows three defect bands, the strongest being the $\mathrm{D}$ band centered at about $1350 \mathrm{~cm}^{-1}$ $[28,29]$. The nonirradiated target foil region exhibits Raman features typical of disordered and amorphous carbon. The irradiated portions of the target foils showed nearly identical features: the ratio of the intensity of the D and $\mathrm{G}$ band was slightly greater (about 3\%) for the irradiated region ( $\mathrm{ID} / \mathrm{IG}=1.28$ and 1.35 for the nonirradiated and irradiated zone, respectively), suggesting a marginal increase in the disordered and amorphous character of the carbon film upon heavy irradiation, but no significant change of structure of the carbon material under beam exposure.

Charged particles are detected in three annular silicon strip detectors covering $30 \%$ of the $4 \pi$ solid angle. For gamma-ray detection, STELLA employs an array of 36 lanthanum bromide $\left[\mathrm{LaBr}_{3}(\mathrm{Ce})\right]$ scintillator detectors from the UK FATIMA Collaboration [30,31], which have high energy resolution $(\approx 3 \%$ at $662 \mathrm{keV})$. The subnanosecond timing of these detectors allows tight time coincidences with charged particles for background reduction as demonstrated in Fig. 2, where the low-level internal radioactivity of the $\mathrm{LaBr}_{3}(\mathrm{Ce})$ material represents the principal background. Such background is associated with the decay 
from both the primordial radionuclide ${ }^{138} \mathrm{La}$ and members of the ${ }^{227}$ Ac decay chain which are incorporated in trace amounts during crystal production [32] leading to a counting rate of the order of $1-10 \mathrm{~Bq}$ per $\mathrm{cm}^{3}$. However, demanding prompt-time coincidences with the evaporated charged particles, as presented in Fig. 2, removes virtually all of this contribution to the background. The observed positions of the 789 and $1436 \mathrm{keV}$ gamma ray peaks from the internal activity were, in fact, used to correct for the temperaturedependent gain drift of the gamma-ray detectors and their associated electronics with a precision of a few $\mathrm{keV}$ [24].

Measurements. - In an initial phase of measurements, data were taken using fixed targets with thicknesses varying from 20 to $70 \mu \mathrm{g} / \mathrm{cm}^{2}$, in the relative-energy range $E_{\text {rel }}=$ 4.5 to $5.5 \mathrm{MeV}$ where cross sections are typically of the order of millibarns. The initial data show good conformity with earlier measurements $[18,19,21,23]$ giving confidence in the present methodology. To study the lowest collision energies, the rotating target mechanism was employed allowing the target foils to sustain a $2 \mathrm{p} \mu \mathrm{A}$ beam for several days without breaking.

The data from the silicon array and $\mathrm{LaBr}_{3}(\mathrm{Ce})$ array were recorded independently onto two digital dataacquisition systems and subsequently merged using correlated time stamps. The excellent timing resolution of the $\mathrm{LaBr}_{3}(\mathrm{Ce})$ as a "start" detector combined with the different effective interaction time of protons and alpha particles within the silicon substrate, automatically provides proton and alpha discrimination using the STELLA digital DAQ, which is a key improvement over earlier coincidence techniques using analogue electronics [24].

For the evaluation of exclusive cross sections to the final systems ${ }^{23} \mathrm{Na}+p$ and ${ }^{20} \mathrm{Ne}+\alpha$, the individual reaction channels $\alpha_{i}$ and $p_{i}$ to the $i$ th excited state were selected by requiring coincidences with the corresponding gamma-ray

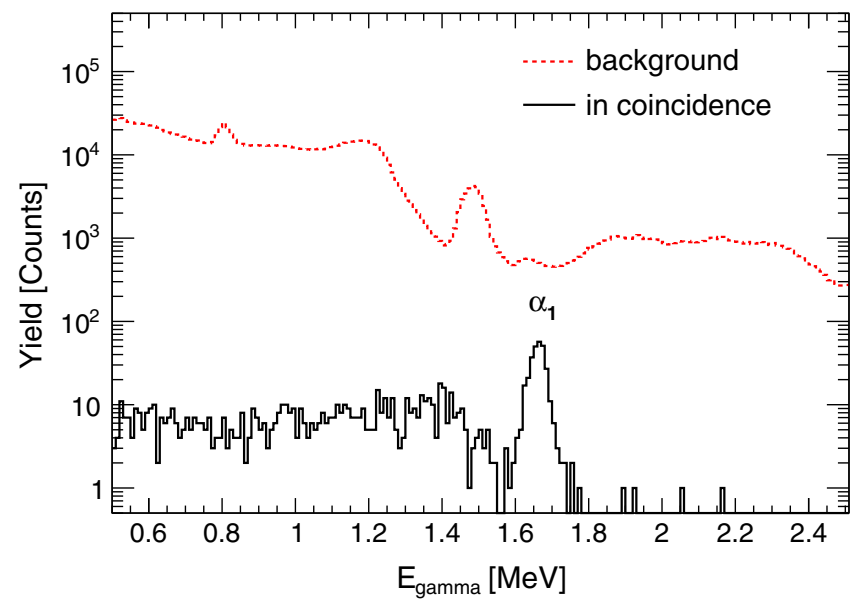

FIG. 2. Gamma-ray spectra obtained for $E_{\text {beam }}=3.83 \mathrm{MeV}$, without coincidence (red dashed showing the internal activity) and in coincidence (black solid) with the alpha particle channel, $\alpha_{1}$. transitions deexciting the states in the daughter nuclei. The principal gamma-ray transitions are those deexciting the first excited states of ${ }^{20} \mathrm{Ne}(1.634 \mathrm{MeV})$ and ${ }^{23} \mathrm{Na}$ $(0.440 \mathrm{MeV})$ which have corresponding gamma-ray detection efficiencies of $2 \%$ and $6 \%$, respectively. Information on the expected branching to different alpha and proton channels from the literature [18], found to be approximately constant in the energy regime considered, has been used in order to correct for reaction channels not observed in the present study including, naturally, the ground-state branches which would not be expected to be seen using a gamma-ray coincidence technique. The systematic uncertainty from averaging amounts to $2 \%$; a $3 \sigma$ confidence interval is adopted for the extrapolation to the lowest energies beyond the available branching ratio data. Even if there were large fluctuations in the branching ratio other than the $3 \sigma$ interval accounted for in the extrapolation, this would be subsumed by the dominant statistical error for the lowest measured data points. For the acceptance corrections, the gamma angular distributions are known to be nearly isotropic $[19,33]$ while charged-particle angular distributions were found to flatten out quickly with decreasing energy $[18,34]$.

Fusion event selection is demonstrated in Fig. 3 for $E_{\text {rel }}=3.77 \mathrm{MeV}$, where the deuterium contamination from $d\left({ }^{12} \mathrm{C}, p\right){ }^{13} \mathrm{C}$ reactions [20] is entirely removed by selecting the $\gamma$-ray transition depopulating the first excited state in ${ }^{20} \mathrm{Ne}$.

For the lowest energies, a statistical analysis was carried out where the background rate in the coincident energy and timing gate is determined under beam conditions. The evaporation products have a well-defined and relatively

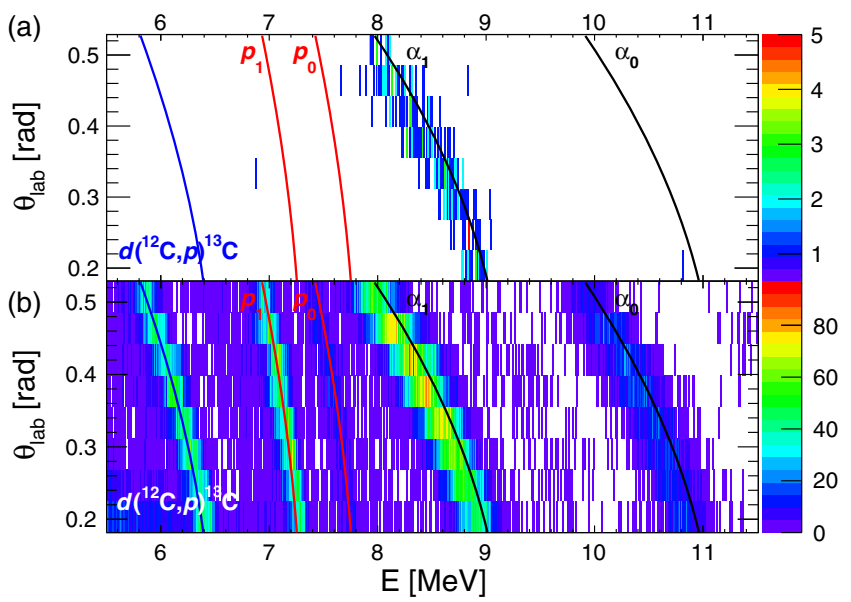

FIG. 3. Charged particle spectra from the ${ }^{12} \mathrm{C}+{ }^{12} \mathrm{C}$ fusion reaction as a function of lab angle for $E_{\text {rel }}=3.77 \mathrm{MeV}$. The bottom spectrum is the singles data without gamma-ray coincidence. The top spectrum is that for alpha-particle events in coincidence with a $1634 \mathrm{keV}$ gamma ray. The locii are shown for different particle groups including protons from the contaminating $d\left({ }^{12} \mathrm{C}, p\right){ }^{13} \mathrm{C}$ reaction. 
narrow time offset with respect to deexcitation gammas, while noncorrelated background was determined well outside this coincidence window applying the same particle selection criteria but wider timing gates for higher statistical relevance. Using the likelihood estimation methods of Feldman [35], one-sigma confidence intervals were obtained around the signal for measured background and observation rates. The procedure is depicted in Fig. 4 for measurements at $E_{\text {rel }}=2.16 \mathrm{MeV}$ with three-sigma bands (dashed lines) around the nominal kinematic locus for the $p_{1}$ proton channel (solid line).

The carbon beam undergoes energy loss predominantly from multiple scattering in the target foils. To calculate the effective beam energy $E_{\text {eff }}$, the varying fusion cross section within the target was interpolated with an exponential response function:

$$
\left.\sigma(E)=\sigma_{s} \frac{E_{s}}{E} \exp \left\{A_{0}\left(E-E_{s}\right)-B_{0} \frac{1}{E_{s}^{N_{p}-1}\left(N_{p}-1\right)}\left[\frac{E_{s}}{E}\right)^{N_{p}-1}-1\right]\right\},
$$

where $\sigma_{s}, E_{s}, A_{0}$, and $B_{0}$ are free parameters and $N_{p}=1.5$ [36]. In the minimization, cross-section data were matched with the response function in the area of each energy loss interval. The effective beam energy was then interpolated as the weighted mean value determined from the cross section drop within the energy-loss interval.

Results.-In order to display the sub-barrier cross sections over a wide energy range, data are commonly expressed in terms of the modified $S$-factor $S^{*}$, where the exponential decrease related to the dominant tunneling

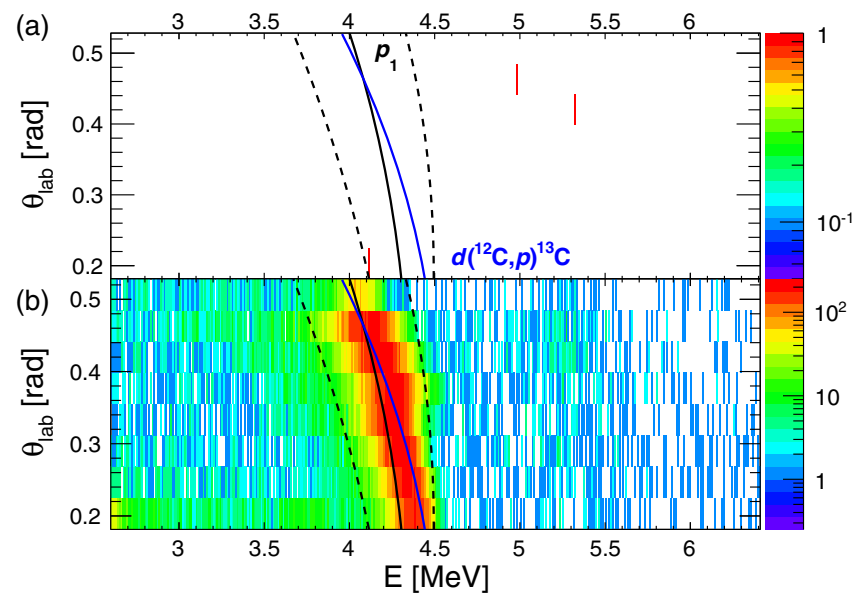

FIG. 4. Charged particle spectra from the ${ }^{12} \mathrm{C}+{ }^{12} \mathrm{C}$ fusion reaction as a function of lab angle for $E_{\text {rel }}=2.16 \mathrm{MeV}$. The bottom spectrum is the singles data without gamma-ray coincidence dominated by protons from the contaminating $d\left({ }^{12} \mathrm{C}, p\right){ }^{13} \mathrm{C}$ reaction (locus labeled in blue). The top spectrum is that for proton events in coincidence with a $440 \mathrm{keV}$ gamma ray. The locus is shown for the $p_{1}$ events with the dashed lines indicating three-sigma windows on the proton energy resolution. effect through the repulsive potential is removed by defining

$$
S^{*}=\sigma E \exp (2 \pi \eta+g E)
$$

where $\eta=Z_{1} Z_{2} e^{2} / \hbar \nu$ is the Sommerfeld parameter and $g=0.122 \sqrt{\mu R^{3} / Z_{1} Z_{2}}$ is the form factor in ${ }^{12} \mathrm{C}+{ }^{12} \mathrm{C}$ reactions derived for $l=0$ states in a square-well model potential $[3,37]$, with the reduced mass $\mu$, the square-well radius $R$, and the charge $Z_{1,2}$ of the nuclei. The correction $\exp (g E)$ is a form factor and needed in the present case as the interaction radius and the energy involved are so large. $S$ factors for ${ }^{12} \mathrm{C}+{ }^{12} \mathrm{C}$ fusion corresponding to alpha evaporation are presented in Fig. 5, and with proton evaporation in Fig. 6 as a function of the relative energy $E_{\text {rel }}$, and compared to previous measurements. Two extrapolations are presented based on a smoothed out average cross section (blue dash-dotted line) [38] and a phenomenological hindrance model (red dotted line) [36]. In the Supplemental Material [39], an excerpt of the energy region of the hindrance phenomenon [40] is provided.

The present data do not require any reaction model for their interpretation (such as in the recent THM experiment) and span the region from the Coulomb barrier down to the upper end of the Gamow window with significantly improved accuracy. They are in good agreement with the data reported by Jiang et al. [23] using similar techniques, and with the renormalized THM data [17]. In the intermediate energy range at $E_{\text {rel }}=3.8 \mathrm{MeV}$, a more prominent resonance is observed compared to previous measurements $[19,20]$.

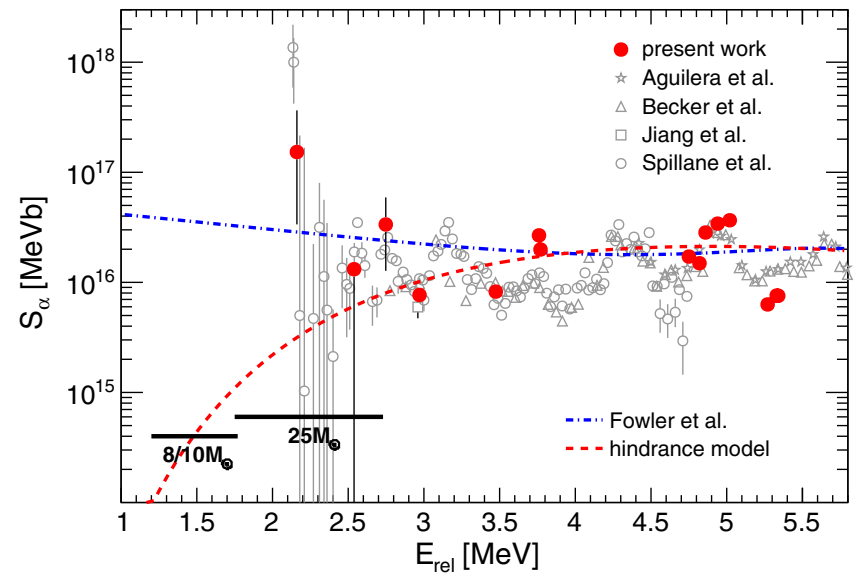

FIG. 5. $S$-factor measurements for the ${ }^{12} \mathrm{C}+{ }^{12} \mathrm{C}$ fusion reaction as a function of $E_{\text {rel }}$ for the final system ${ }^{20} \mathrm{Ne}+\alpha$. The present data, at the effective beam energy (see text for details), are presented as red circles and compared to earlier measurements (gray symbols) [18,19,21,23]. The blue dash-dotted line is a standard extrapolation of the data [38], while the red dashed line corresponds to a sub-barrier hindrance model [36]. The Gamow windows for $8 / 10 M_{\odot}$ and $25 M_{\odot}$ correspond to stellar temperatures of 0.5 and $0.9 \mathrm{GK}$, respectively. 


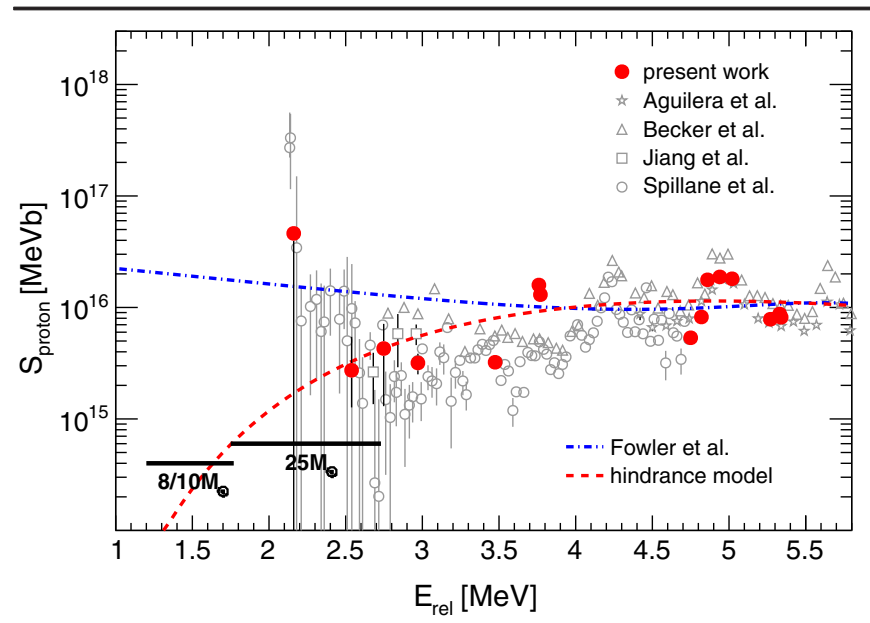

FIG. 6. $S$-factor measurements in similar context as Fig. 5, but for the final system ${ }^{23} \mathrm{Na}+p$.

The data for the proton-evaporation channel strongly supports the extrapolation based on the hindrance model with an excellent match well below the predicted $S$-factor maximum around 3 to $4 \mathrm{MeV}$ [40]; the lowest data point being less discriminating since it corresponds to a onesigma upper limit. The data for the alpha-evaporation channel broadly support the hindrance model and also provide evidence for the resonance at $E_{\text {rel }}=2.14 \mathrm{MeV}$ reported by Spillane et al. [19], albeit with a reduced resonance strength.

Corrections from electron screening effects can lead to an enhancement of the reaction rate determined in the laboratory for its application in the astrophysical site. The nuclei in the target material are shielded by the surrounding electrons, which effectively lowers the Coulomb well as compared to a bare nucleus. Therefore an enhanced cross section is detected. This screening effect on the $S$ factor is most efficient at the lowest beam energy and in the present study it was estimated based on the Rutherford-Bohr model of the atom [41], where the shielding potential is defined by the Coulomb field at the innermost electron ring. The effect for ${ }^{12} \mathrm{C}+{ }^{12} \mathrm{C}$ collisions considered here is found to be less than $3 \%$, which is included in the systematic uncertainty $(\leq 17 \%)$. Note that in experimental studies of reactions with light projectiles $(Z \leq 3)$ a systematically higher enhancement was observed [42], while a more complementary analysis procedure [43] could extract screening potentials as predicted with the atom-physics model.

Discussion.-The measurement with the STELLA experimental station has allowed the extraction of reliable excitation functions for the ${ }^{12} \mathrm{C}+{ }^{12} \mathrm{C}$ fusion reaction over 8 orders of magnitude in cross section. It was possible to probe well below the Coulomb barrier into the Gamow window for massive stars using the gamma-particle coincidence technique. Key to these advances were the excellent timing properties of the $\mathrm{LaBr}_{3}$ detectors, the novel self-supporting rotating target system, and the dedicated low-noise particle detector system.

The results identify three distinct regimes in the subCoulomb range: (i) The moderate sub-barrier regime above $E_{\text {rel }}=4.5 \mathrm{MeV}$ - where it has been possible to unambiguously validate our experimental concept by accurately measuring the excitation function of the ${ }^{12} \mathrm{C}+{ }^{12} \mathrm{C}$ fusion reaction. (ii) The deep-sub-barrier regime from $E_{\text {rel }}=2.5$ to $4 \mathrm{MeV}$-where the Fowler standard extrapolation systematically overestimates the results and where hindrance is observed. (iii) The 25 solar masses Gamow window-below $E_{\text {rel }}=2.5 \mathrm{MeV}$-where the $S$ factor rises up and may indicate a change in the fusion mechanism. The latter may reveal either the presence of a resonance and/or may be interpreted as the consequence of the low level density of states in ${ }^{24} \mathrm{Mg}$ at these excitation energies [13]. The observation of hindrance behavior, as the dominant mechanism with molecular doorway resonances built on it, is a plausible explanation but this would artificially split the fusion process into two processes. A simpler explanation could be that the large spacing of narrow levels in the eveneven ${ }^{24} \mathrm{Mg}$ nucleus, accessed via the collision of the two identical bosons $\left({ }^{12} \mathrm{C}\right.$ nuclei), has a limited phase space (akin to Pauli repulsion), where only a few final states are available for the fusion process to occur.

The fusion-hindrance behavior suggested by the present work would serve to raise the carbon-burning ignition temperature since it shifts the equilibrium related to heat generation by ${ }^{12} \mathrm{C}$ fusion, and that of the temperature and density-dependent heat transport by neutrinos [44]. As a consequence, the lowest fusion energy explored in the present work would be well inside the Gamow window for a star of 25 solar masses. In addition, neutron-seed generation should be affected by the observed fusion trend $[45,46]$. Our results will be applied to further investigations of the advanced burning phases in massive stars, to improve our understanding of their end points and the flux of neutrinos produced as well as providing information on the probability of type Ia supernovae $[47,48]$.

We thank M. Lorrigiola (LNL, Padova, Italy) and G. Frémont (GANIL, Caen, France) for the excellent preparation of the reaction targets. The construction of STELLA was funded by the University of Strasbourg IdEX program and CNRS Strasbourg. The Andromède facility (ANR-10EQPX- 23) was funded by the program for future investment, EQUIPEX. This work was also partially supported by the UK Science and Technology Facility Council via Grants No. ST/L005743/1, No. ST/P003885/1, and No. ST/P005314/1. P. H. R. and R. S. acknowledge support from the UK Department for Business, Energy and Industrial Strategy (BEIS) via funding for the UK National Measurement System. D. G. J. and S. C. acknowledge support from their Fellowships in the University of Strasbourg Institute of Advanced Study (USIAS). 
*Corresponding author.

Sandrine.Courtin@iphc.cnrs.fr

${ }^{\dagger}$ Marcel.Heine@iphc.cnrs.fr

[1] S. E. Woosley, A. Heger, and T. A. Weaver, Rev. Mod. Phys. 74, 1015 (2002).

[2] H. Reeves and E. E. Salpeter, Phys. Rev. 116, 1505 (1959).

[3] C. E. Rolfs and W. S. Rodney, Cauldrons in the Cosmos, 1st ed. (University of Chicago Press, Chicago, 1988).

[4] A. Cumming and L. Bildsten, Astrophys. J. 559, L127 (2001).

[5] D. A. Bromley, J. A. Kuehner, and E. Almqvist, Phys. Rev. Lett. 4, 365 (1960).

[6] K. A. Erb, R. R. Bettsand, S. K. Korotkyand, M. M. Hindiand, P. P. Tungand, M. W. Sachsand, S. J. Willett, and D. A. Bromley, Phys. Rev. C 22, 507 (1980).

[7] M. Freer, H. Horiuchi, Y. Kanada-Enyo, D. Lee, and Ulf-G. Meißner, Rev. Mod. Phys. 90, 035004 (2018).

[8] J.-P. Ebran, E. Khan, T. Nikšić, and D. Vretenar, Phys. Rev. C 90, 054329 (2014).

[9] Y. Kanada-Enyo, Prog. Theor. Phys. 117, 655 (2007).

[10] E. Epelbaum, H. Krebs, T.-A. Lähde, D. Lee, and Ulf-G. Meißner, Phys. Rev. Lett. 109, 252501 (2012).

[11] M. Kimura and Y. Chiba, J. Phys. Conf. Ser. 569, 012005 (2014).

[12] A. Diaz-Torres and M. Wiescher, Phys. Rev. C 97, 055802 (2018).

[13] C. L. Jiang, B. B. Back, H. Esbensen, R. V. F. Janssens, K. E. Rehm, and R. J. Charity, Phys. Rev. Lett. 110, 072701 (2013).

[14] C. L. Jiang et al., Phys. Rev. Lett. 89, 052701 (2002).

[15] B. B. Back, H. Esbensen, C. L. Jiang, and K. E. Rehm, Rev. Mod. Phys. 86, 317 (2014).

[16] A. Tumino et al., Nature (London) 557, 687 (2018).

[17] A. M. Mukhamedzhanov, D. Y. Pang, and A. S. Kadyrov, Phys. Rev. C 99, 064618 (2019).

[18] H. W. Becker, K. U. Kettner, C. Rolfs, and H. P. Trautvetter, Z. Phys. A 303, 305 (1981).

[19] T. Spillane et al., Phys. Rev. Lett. 98, 122501 (2007).

[20] J. Zickefoose et al., Phys. Rev. C 97, 065806 (2018).

[21] E. F. Aguilera, P. Rosales, E. Martinez-Quiroz, G. Murillo, and M. C. Fernández, Nucl. Instrum. Methods Phys. Res., Sect. B 244, 427 (2006).

[22] C. L. Jiang et al., Nucl. Instrum. Methods Phys. Res., Sect. B 682, 12 (2012).

[23] C. L. Jiang et al., Phys. Rev. C 97, 012801(R) (2018).

[24] M. Heine et al., Nucl. Instrum. Methods Phys. Res., Sect. A 903, 1 (2018).

[25] S. Della-Negra, Innovation Rev. 93, 38 (2016).
[26] M. J. F. Healy, Nucl. Instrum. Methods Phys. Res., Sect. B 129, 130 (1997).

[27] A. C. Ferrari and J. Robertson, Phys. Rev. B 61, 14095 (2000).

[28] A. Sadezky, H. Muckenhuber, H. Grothe, R. Niessner, and U. Pöschl, Carbon 43, 1731 (2005).

[29] E. Charon, J. N. Rouzaud, and J. Aleon, Carbon 66, 178 (2014).

[30] O. J. Roberts, A. M. Bruce, P. H. Regan, Z. Podolyák, C. M. Townsley, J. F. Smith, K. F. Mulholland, and A. Smith, Nucl. Instrum. Methods Phys. Res., Sect. A 748, 91 (2014).

[31] P. H. Regan, Radiat. Phys. Chem. 116, 38 (2015).

[32] F. G. A. Quarati, I. V. Khodyuk, C. W. E. van Eijk, P. Quarati, and P. Dorenbos, Nucl. Instrum. Methods Phys. Res., Sect. A 683, 46 (2012).

[33] K. U. Kettner, H. Lorenz-Wirzba, and C. Rolls, Z. Phys. A 298, 65 (1980).

[34] M. G. Mazarakis and W. E. Stephens, Phys. Rev. C 7, 1280 (1973).

[35] G. J. Feldman and R. D. Cousins, Phys. Rev. D 57, 3873 (1998).

[36] C. L. Jiang, K. E. Rehm, B. B. Back, and R. V. F. Janssens, Phys. Rev. C 79, 044601 (2009).

[37] J. R. Patterson, H. Winkler, and C. S. Zaidins, Astrophys. J. 157, 367 (1969).

[38] W.-A. Fowler, G.-R. Caughlan, and B.-A. Zimmerman, Annu. Rev. Astron. Astrophys. 13, 69 (1975).

[39] See Supplemental Material at http://link.aps.org/ supplemental/10.1103/PhysRevLett.124.192701 for extended data display.

[40] C. L. Jiang, K. E. Rehm, B. B. Back, and R. V. F. Janssens, Phys. Rev. C 75, 015803 (2007).

[41] H. J. Assenbaum, K. Langanke, and C. Rolfs, Z. Phys. A 327, 461 (1987).

[42] G. Fiorentini, R. W. Kavanagh, and C. Rolfs, Z. Phys. A 350, 289 (1995).

[43] F. C. Barker, Nucl. Phys. A707, 277 (2002).

[44] M. E. Bennett et al., Mon. Not. R. Astron. Soc. 420, 3047 (2012).

[45] M. Pignatari, R. Hirschi, M. Wiescher, R. Gallino, M. Bennett, M. Beard, C. Fryer, F. Herwig, G. Rockefeller, and F. X. Timmes, Astrophys. J. 762, 31 (2013).

[46] L. R. Gasques, E. F. Brown, A. Chieffi, C. L. Jiang, M. Limongi, C. Rolfs, M. Wiescher, and D. G. Yakovlev, Phys. Rev. C 76, 035802 (2007).

[47] O. Straniero, L. Piersanti, and S. Cristallo, J. Phys. Conf. Ser. 665, 012008 (2016).

[48] M. Limongi and A. Chieffi, Astrophys. J. 647, 483 (2006). 